Development and performance of Microbulk Micromegas detectors

This article has been downloaded from IOPscience. Please scroll down to see the full text article.

2010 JINST 5 P02001

(http://iopscience.iop.org/1748-0221/5/02/P02001)

View the table of contents for this issue, or go to the journal homepage for more

Download details:

IP Address: 137.138.124.14

The article was downloaded on 11/08/2010 at 13:53

Please note that terms and conditions apply. 
$1^{\text {st }}$ International Conference on Micro Pattern Gaseous Detectors, JUNE 12-15, 2009

Kolympari, Crete, Greece

\section{Development and performance of Microbulk Micromegas detectors}

S. Andriamonje, ${ }^{a}$ D. Attie,${ }^{b}$ E. Berthoumieux,${ }^{b}$ M. Calviani, ${ }^{a}$ P. Colas,${ }^{b}$ T. Dafni,${ }^{c}$

G. Fanourakis, ${ }^{d}$ E. Ferrer-Ribas,${ }^{b}$ J. Galan, ${ }^{c}$ T. Geralis, ${ }^{d}$ A. Giganon, ${ }^{b}$ I. Giomataris,${ }^{b}$ A. Gris, ${ }^{a}$ C. Guerrero Sanchez, ${ }^{a}$ F. Gunsing, ${ }^{b}$ F.J. Iguaz, ${ }^{c}$ I. Irastorza, ${ }^{c}$

R. De Oliveira, ${ }^{a}$ T. Papaevangelou ${ }^{b, 1}$ J. Ruz, ${ }^{a}{ }^{\text {I. Savvidis, }}{ }^{e}$ A. Teixera ${ }^{a}$ and A. Tomás ${ }^{c}$

${ }^{a}$ CERN, European Organization for Particle Physics and Nuclear Research, Geneva, Switzerland

${ }^{b}$ IRFU, Centre d'Etudes Nucléaires de Saclay, Gif sur Yvette, France

${ }^{c}$ Laboratorio de Física Nuclear y Astropoartículas, Universidad de Zaragoza, Zaragoza, Spain

${ }^{d}$ Institute of Nuclear Physics, NCSR Demokritos, Athens, Greece

${ }^{e}$ Aristotle University,

Thessaloniki, Greece

E-mail: Thomas.Papaevangelou@cern.ch

ABSTRACT: A new Micromegas manufacturing technique, based on kapton etching technology, has been developed recently, resulting in further improvement of the characteristics of the detector, such as uniformity and stability. Excellent energy resolution has been obtained, reaching $11 \%$ FWHM for the $5.9 \mathrm{keV}$ photon peak of the ${ }^{55} \mathrm{Fe} \mathrm{X}$-ray source and $1.8 \%$ FWHM (with possible evidence of less than $1 \%$ ) for the $5.5 \mathrm{MeV}$ alpha peak of the ${ }^{241} \mathrm{Am}$ source. The new Microbulk detector shows several advantages like flexible structure, low material and high radio-purity, opening thus new possibilities for both accelerator and low counting-rate experiments. The detector has already been used in CAST and n-TOF, while it is being tested for future neutrinoless double-beta decay experiments like NEXT. Details of the production of several types of Microbulk detectors will be described. First benchmark results will be presented, demonstrating the enhanced performance of Microbulk detectors.

KEYWORDS: Gaseous detectors; Micropattern gaseous detectors (MSGC, GEM, THGEM, RETHGEM, MICROMEGAS, InGrid, etc); X-ray detectors; Neutron detectors (cold, thermal, fast neutrons)

\footnotetext{
${ }^{1}$ Corresponding author.
} 


\section{Contents}

1 Introduction 1

2 Manufacturing process $\quad 2$

3 Characterization measurements 3

4 Performance in physics experiments 5

4.1 Microbulk in CAST 5

4.2 Microbulk for NEXT 8

4.3 Microbulk in $\mathrm{n}_{-} \mathrm{TOF} \quad 8$

5 Future developments 9

6 Conclusion 10

\section{Introduction}

Gas proportional counters based on the Micromegas [1,2] technology are an example of a new generation of detectors that exploit narrow anode-cathode gaps, rather than thin wires, to create gas gain. These detectors, called Micropattern Gaseous Detectors, are inherently pixel detectors that can be made at large size for reasonable costs. Because of their intrinsic gain and roomtemperature operation, they can be instrumented at very low power per unit area, making them valuable for a variety of particle physics applications where large-area particle tracking is required. Micromegas is a Parallel Plate Detector (PPD) with three electrodes, cathode, micromesh and anode and a narrow amplification space, typically 50-100 microns, between the micromesh and the anode. The detector gain depends directly on the distance between the micromesh and anode, therefore controlling the geometry of the amplification region is crucial in order to achieve good energy resolution [2].

In its traditional form, Micromegas is built by use of a thin electroformed Nickel mesh as the Frisch grid for dividing the gas chamber into the drift and amplification gaps. The mesh is stretched and glued on a removable glass-fiber frame and placed above the anode plane [3]. To maintain a uniform gap, small cylindrical insulating spacers made of photo-imageable resin, $100 \mu \mathrm{m}$ thick and $150 \mu \mathrm{m}$ in diameter, are fixed to the anode strips by a standard printed circuit technique. Another type of mesh based on simple chemical-etching techniques on a single foil of kapton copper plated on both sides has been also developed. The manufacturing process relies on the high accuracy of the photolithography technique that allows to print on a $5 \mu \mathrm{m}$ copper grid with $25 \mu \mathrm{m}$ openings and a pitch of $50 \mu \mathrm{m}$; the kapton is partially removed, leaving kapton pillars that are used as spacers for the amplification gap of the detector [4]. 


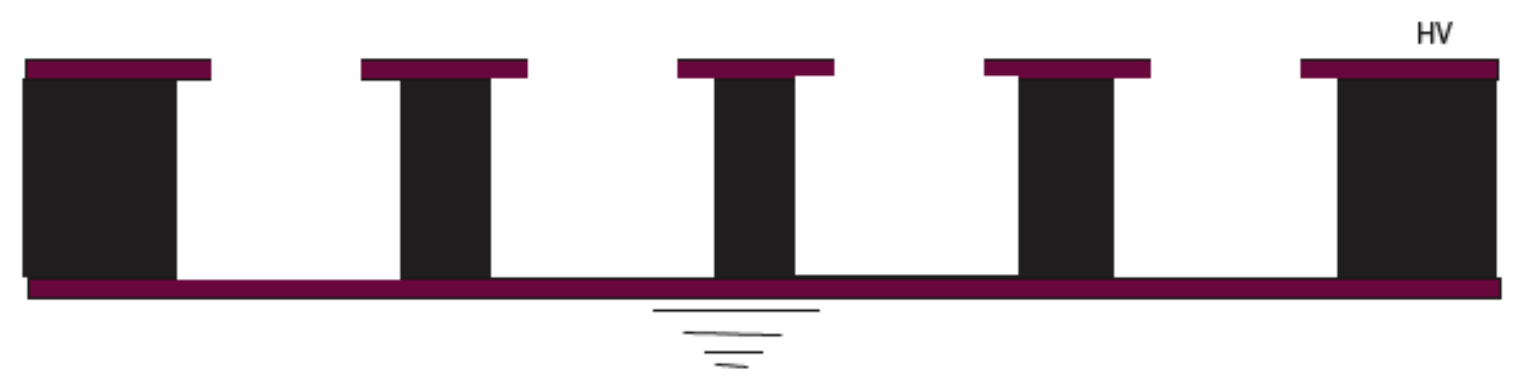

Figure 1. Kapton pillars are created below the copper in each mesh step.

Efforts have been focused on producing the amplification region as a single piece using the newly developed "bulk" method [5]. A woven mesh is laminated on a PC board covered by a photoimageable polyimide film, and the pillars are made by a photochemical technique with insulation through the grid. Such an 'all-in-one' detector, called 'bulk' Micromegas, is robust and will allow large areas to be made in one piece. This industrial assembly process allows the regular production of large, stable and inexpensive detector modules [5].

We present a novel fabrication technique using kapton thin-foil etching technology. We have used this technique to fabricate detectors, reading out anode and cathode strips, in a single integrated structure. We present gain and energy resolution measurements, detailed results stability and uniformity from these detectors, as well as several applications in high energy and nuclear physics.

\section{Manufacturing process}

The raw material is a thin flexible polyimide foil with a thin copper layer on each side. The foil is usually glued on top of a rigid substrate that provides the support of the micro-structure and most of the time carries anode strips or pixels. In some applications demanding low detector material the foil is stretched and glued on a suitable frame.

We have used two slightly different technologies that are described below. In both technologies (see figure 1-2) a standard lithography and kapton etching is used. This technology is inspired by the GEM detector fabrication process invented at CERN [6]. A thin photoresistive film is laminated on top of the kapton foil and it is insolated by UV light to produce the required mask. The copper is then removed by a standard lithographic process, the non-insulated places producing a pattern of a thin mesh. In our first prototype the holes are $30 \mu \mathrm{m}$ in diameter and a pitch of $80 \mu \mathrm{m}$. The polyimide is then etched and partially removed in order to create tiny pillars in the shadow part of the mesh below the copper mesh.

In the second approach the polyimide is completely removed except the points where small pillars are created (about $100 \mu \mathrm{m}$ in diameter) with a pitch of $\sim 1 \mathrm{~mm}$. To achieve this, an additional insolating spot (about $200 \mu \mathrm{m}$ ) is formed during insolation process, leaving after the lithographic process a copper spot of $200 \mu \mathrm{m}$ in diameter (see figure 2). By increasing the duration of the etching process, the polyimide under the mesh is removed completely except the points below the shadow of these copper spots. 


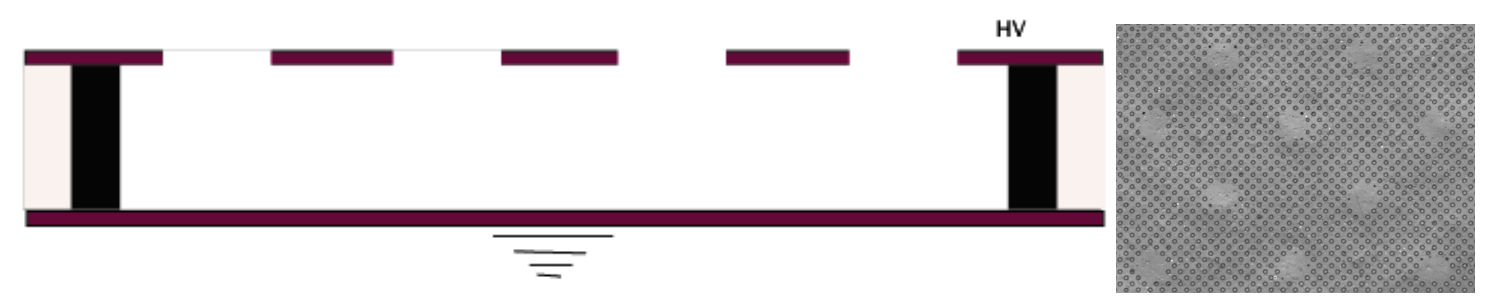

Figure 2. Left: Kapton pillars are created with a step of about 500 microns; Right: photo of a mesh with copper spots used to protect the polyimide bellow during etching in order to form the pillars.

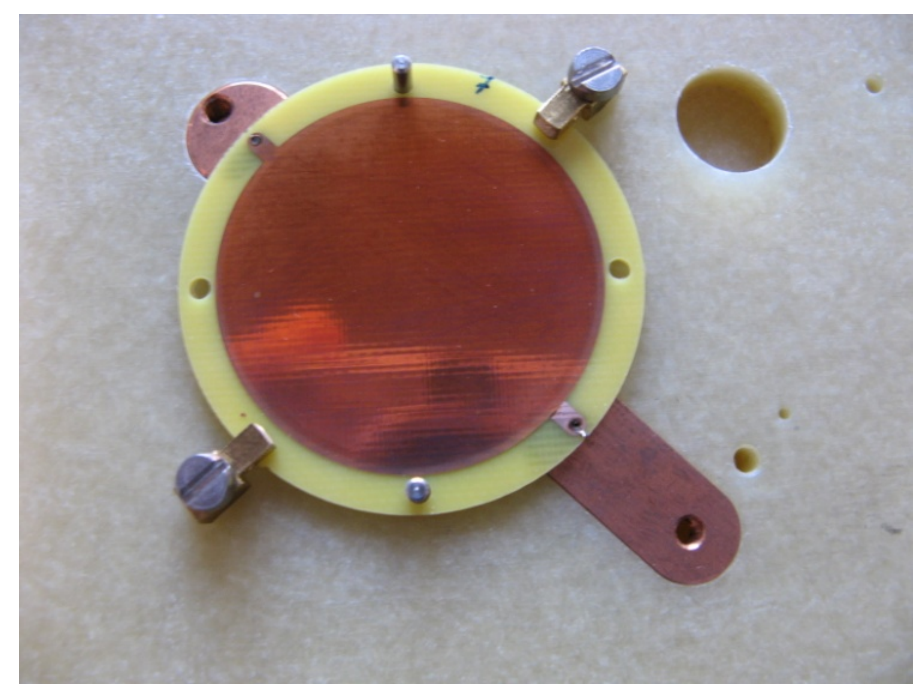

Figure 3. Photo of the $3.5 \mathrm{~cm}$ diameter circular Micromegas.

In both cases a structure of Micromegas is produced with two types of pillars that are maintaining flat the amplification gap between the mesh (cathode) and anode plane. Notice that the pillars are always on the shadow of copper pads therefore any dead space is avoided, as in the conventional structure. This is also an advantage in imaging applications with $\mathrm{x}$-rays or neutrons. We have successfully built prototypes using polyimide foils of 25 and 50 micron thick providing amplification gaps of 25 and 50 micron respectively.

\section{Characterization measurements}

Different batches of $3.5 \mathrm{~cm}$ diameter circular Micromegas were produced at CERN. The amplification gap was $50 \mu \mathrm{m}$ while the anode was non-segmented. A picture of the readout plane can be seen in figure 3. The hole diameters of the mesh are $30 \mu \mathrm{m}$ with a pitch of $100 \mu \mathrm{m}$. These detectors were tested in a test chamber at atmospheric pressure with a gas mixture of argon with 5\% isobutene, having a drift gap of $5 \mathrm{~mm}$. The signal was read out from the Micromegas mesh using an ORTEC 142B preamplifier and fed into an ORTEC 572 shaper amplifier. A multi-channel analyzer AMPTEK MCA-8000A was used for spectra recording.

The energy resolution was measured by irradiating the detector with a ${ }^{55} \mathrm{Fe}$ collimated source. We obtain a result $11.2 \% \mathrm{FWHM}$ at $5.9 \mathrm{keV}$, and a well separated escape peak at $3 \mathrm{keV}$ as shown in 

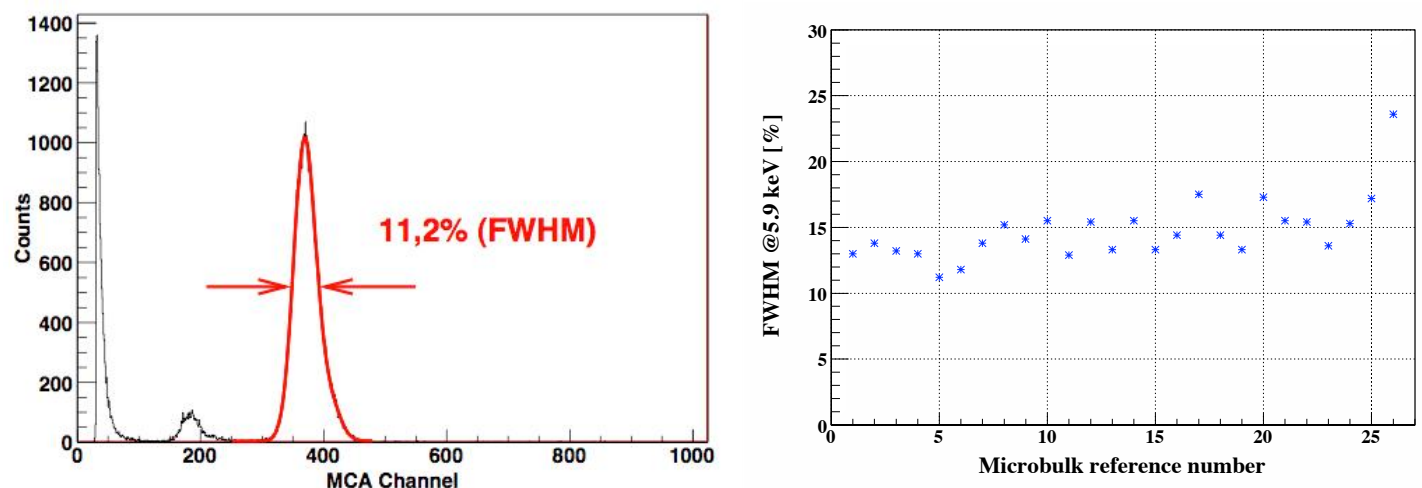

Figure 4. Left: Energy resolution with a ${ }^{55} \mathrm{Fe}$ source. Right: Energy resolution (FWHM) obtained for 26 Microbulk detectors at $5.9 \mathrm{keV}$.

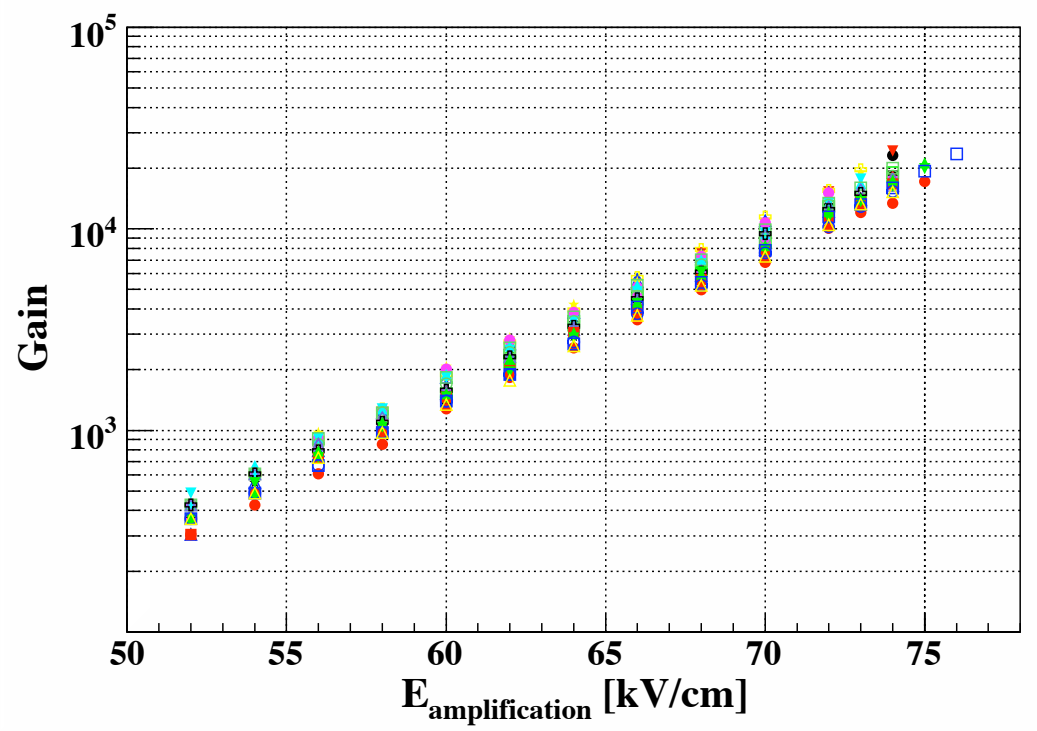

Figure 5. Gain as a function of amplication field in an argon - 5\% isobutane mixture at atmospheric pressure for 26 Microbulk detectors.

figure 4 (Left). Figure 4 (Right) shows the energy resolution for a bunch of 26 Microbulk detectors with a mean value of $15 \%$ FWHM. This value shows an improvement with respect to traditional Micromegas detectors thanks to the better controlled gap size.

Figure 5 shows the gain curve as a function of the amplification field for 26 Microbulks produced over more than 1 year. The gains obtained, reaching values higher than $10^{4}$, are of the same order as in standard Micromegas detectors and the dispersion at a given field value is around $13 \%$. This dispersion accounts, apart from the dispersion in performance, for a variation of environmental conditions as the measurements were realized over one year in a non-controlled pressure and temperature environment.

Microbulks of gap size of $25 \mu \mathrm{m}$ have also been manufactured. Their performance in terms of gain and in comparison with standard Microbulk (50 $\mu \mathrm{m}$ gap size) is given in figure 6 at atmo- 


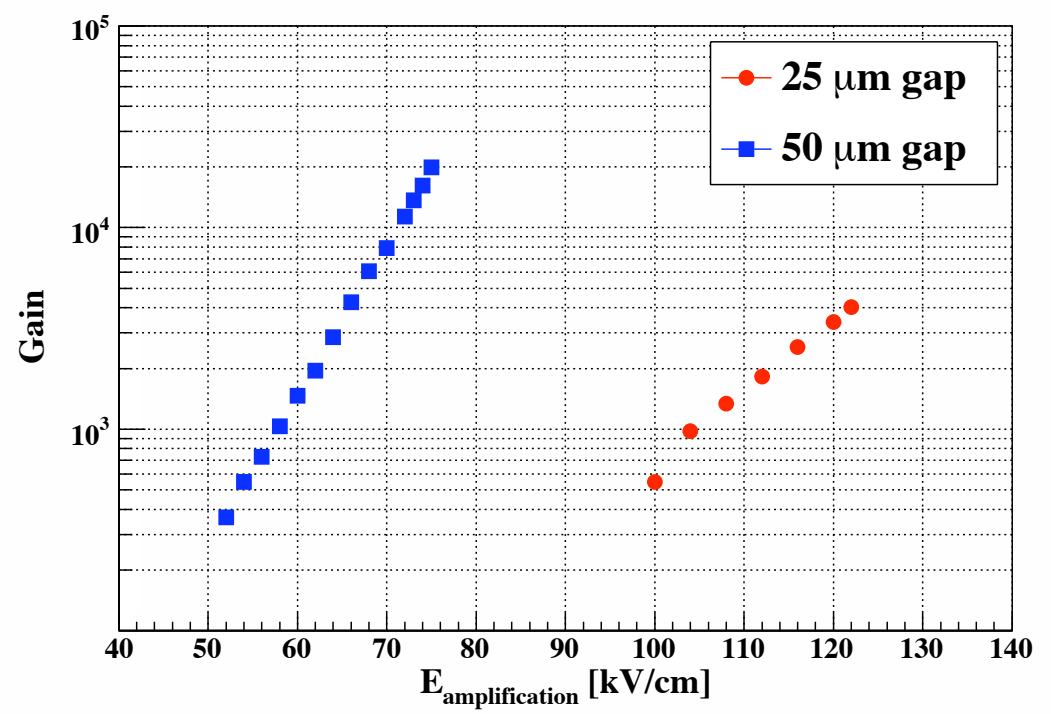

Figure 6. Gain as a function of amplication field in an argon - 5\% isobutane mixture at atmospheric pressure for a Microbulk with a gap of $50 \mu \mathrm{m}$ and $25 \mu \mathrm{m}$.

spheric pressure. The maximum gain obtained with the $25 \mu \mathrm{m}$ gap is not optimized as it is known that smaller gap sizes are best suited for higher pressures. For the $25 \mu \mathrm{m}$ gap size, the optimum is around 2 bar.

The resolution of Microbulk Micromegas detectors at high pressures with high energy particles has been largely studied in reference $[7,8]$ in regard to a high pressure TPC for a future double beta experiment. Here we will only stress two aspects. The first one is that energy resolutions down to $1.8 \%$ and $2 \%$ FWHM have been obtained at $5.5 \mathrm{MeV}$ by using an ${ }^{241} \mathrm{Am}$ alpha source in $2 \%$ and 5\% argon-isobutane mixtures. The second one is that, in pure xenon gas, energy resolutions of $2.8 \%$ and $4.5 \%$ FWHM have been obtained for 2 bar and 4 bar respectively although being limited by gas purity. These preliminary results in pure xenon are very encouraging in the prospects of using Microbulk Micromegas detectors for a double beta decay experiment.

\section{Performance in physics experiments}

Micromegas detectors constructed with Microbulk technology have several advantages like low material, high radiopurity and better energy resolution which makes them very attractive for rare event detection experiments. The detector has been already used for axion searches in CAST [912] and it is being tested for future experiments like NEXT [13]. Furthermore, the minimal amount of material, and the small neutron sensitivity makes these detectors suitable for neutron beam experiments, such as $\mathrm{n}_{-} \mathrm{TOF}$ where Microbulks are being used for beam monitoring.

\subsection{Microbulk in CAST}

The expected signal in CAST is an excess of X-rays of energy 1-10 keV during solar tracking, over the background. Thus, low background detectors are a key element for the experiment's sensitivity. 


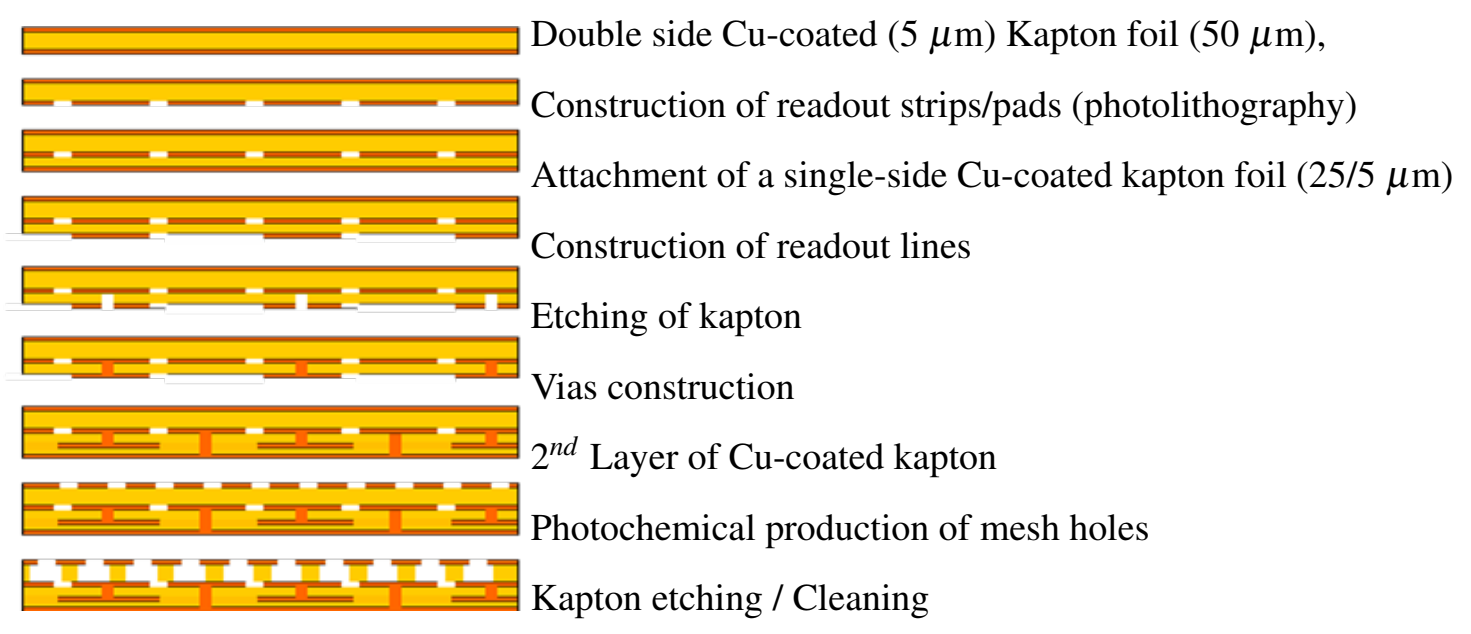

Figure 7. Manufacturing process of a Microbulk with a 2D readout scheme.

Such detectors should show low intrinsic radioactivity, good energy and spatial resolution, time stability and particle discrimination capability. Since 2008 three Microbulk detectors are being used, replacing the conventional Micromegas and the TPC.

The manufacturing process of the CAST Microbulk with a 2D readout scheme is summarized in figure 7. The detector anode consists of square pads interconnected in the diagonal direction through vias in two extra back-layers. In a recent development, the connections in one direction were done at the pads level, so only a single extra layer was needed for the other direction, thus further reducing the detectors material (figure 8 ). The micromesh is formed by holes constructed on top of the pads, insuring in combination with the electron diffusion in the drift gas, that similar amount of charge is deposited on both $\mathrm{X}$ and $\mathrm{Y}$ pads. The strip pitch is $550 \mu \mathrm{m}$, while the spatial resolution is better than $100 \mu \mathrm{m}$ due to the diffusion, which results on a mean multiplicity of $\sim 8$ in each direction for $6 \mathrm{keV} \mathrm{X}$-ray events.

The rest of the detector chamber consists of Plexiglas, while a thin $(5 \mu \mathrm{m})$ aluminized mylar window with an aluminum strong back is used for the coupling with the magnet vacuum. The readout lines are constructed on the same kapton and the front-end electronics are placed well away from the sensitive area, so that they can be placed outside the shielding. All these result in having only very low radioactivity materials (kapton, copper, Plexiglas and aluminum) on the detector area, which in combination with appropriate shielding can lead to a minimal internal background level.

The main source of background in CAST conditions is cosmic muons which give a trigger rate of the order of $1 \mathrm{~Hz}$ in each Micromegas detector. However this background can be suppressed by applying a pattern recognition algorithm on the offline data, since muons have a completely different signature than X-rays of few $\mathrm{keV}$. Another source is environmental radiation like gammas and neutrons, which can be reduced using a passive shielding. In CAST, we are using a shielding that consists of $2.5 \mathrm{~cm}$ of archaeological lead, surrounded by polyethylene of a thickness varying up to $25 \mathrm{~cm}$, limited only by space constrains. Between the lead and the polyethylene there is a 2 $\mathrm{mm}$ thick cadmium foil to absorb thermal neutrons. Inside the lead there is a $5 \mathrm{~mm}$ thick copper layer, also serving as faraday cage. The front-end electronics are placed outside the shielding. The shielding interior is flushed with nitrogen in order to reduce radon radioactivity. 


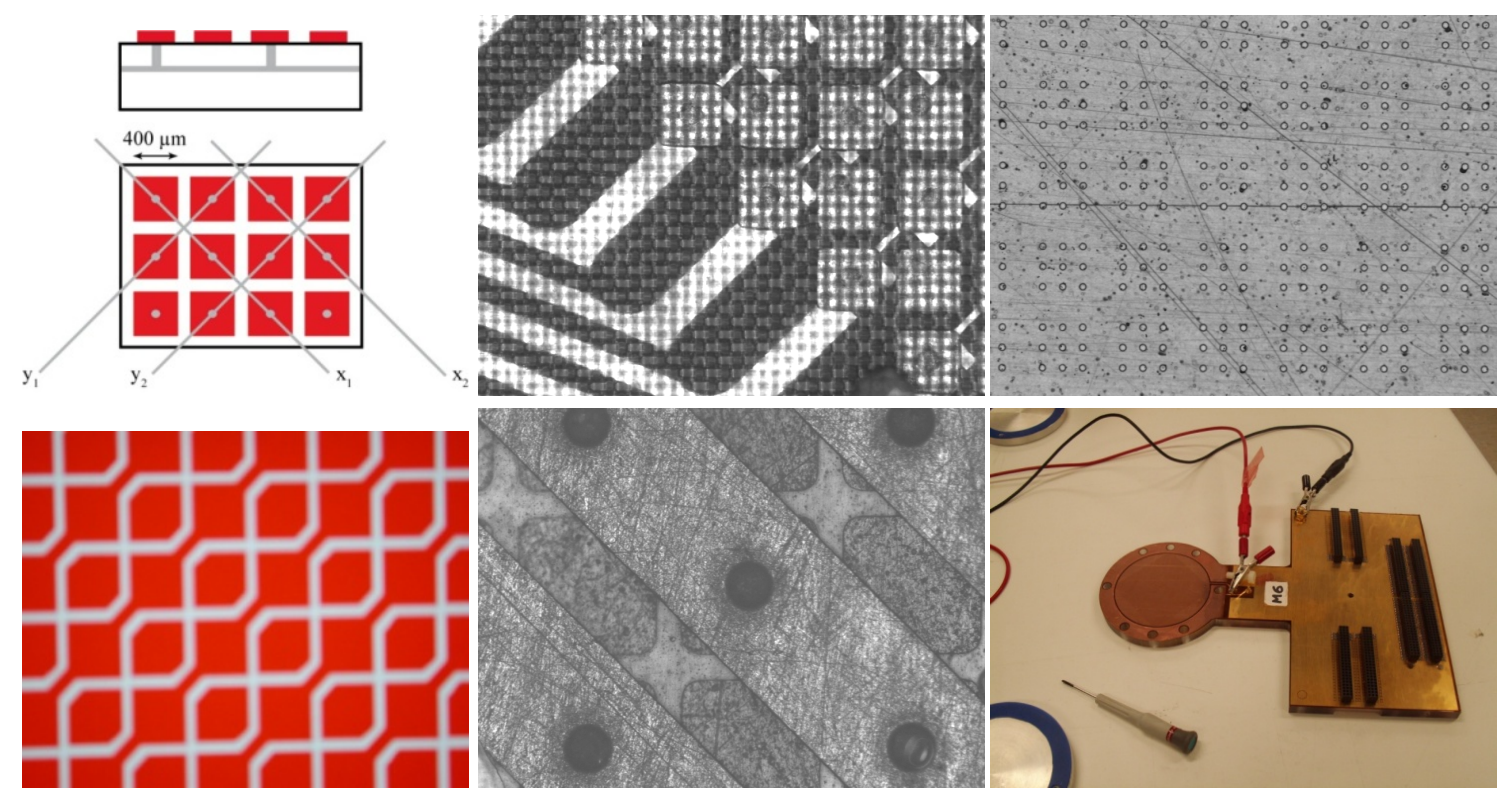

Figure 8. Left: schematic drawing of the $2 \mathrm{D}$ readout schemes, with two extra layer vias (top) and with one direction connected on the pad layer (bottom). Middle: corresponding photos from the front (top) and or the back side (bottom). Right (top): photo of the mesh. Right (bottom): photo of an open detector.
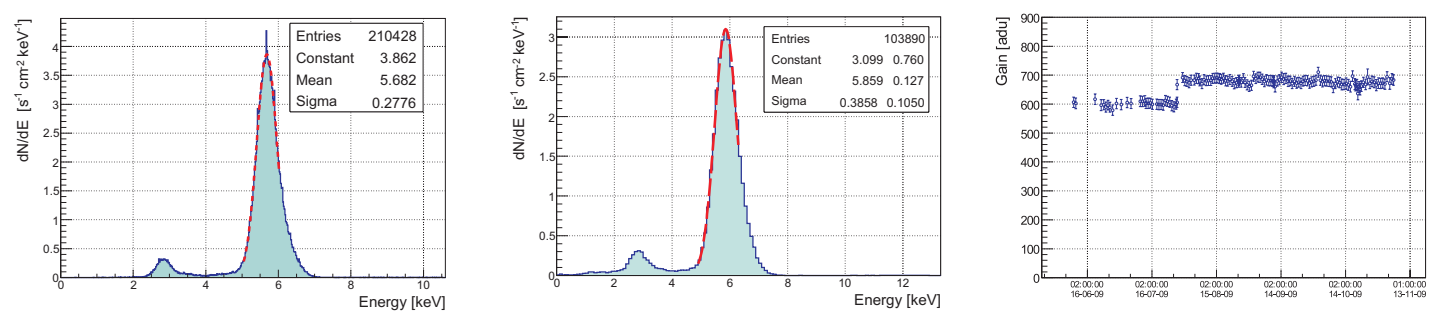

Figure 9. From left to right: 55 Fe energy spectrum with $5 \%$ isobutane collimated source; $2.3 \%$ isobutane non-collimated source; gain stability with time, the steep is due to the increase in the applied voltage by 1 Volt.

In such a configuration the achieved background level is determined by the detector's radiopurity and particle discrimination efficiency, for the same shielding quality. A Microbulk detector, besides its radiopurity, offers better energy resolution and time stability compared to conventional and bulk Micromegas, which lead to enhanced particle discrimination efficiency.

The energy resolution of the CAST Microbulk reaches $11.5 \%$ FWHM at $6 \mathrm{keV}$, for a gas mixture of argon 95\% - isobutane 5\% when the detector is irradiated with a collimated $(\emptyset 15 \mathrm{~mm}){ }^{55} \mathrm{Fe}$ source (figure 9). In CAST, safety rules limit the isobutane fraction to less than $2.3 \%$, so the resolution is relatively reduced, however it still reaches $15.5 \%$ for non-collimated total surface irradiation. This performance is comparable with the one achieved with the single-pad Microbulk detectors used in laboratory tests, showing that the $2 \mathrm{D}$ structure does not affect the energy resolution.

The stability of the detector with time is also crucial in a rare event detection experiment, where statistics are accumulated over long periods. The gain of Microbulk detectors is more stable 
than traditional Micromegas due to the attachment of the mesh to the anode. Microbulks are also less sensitive to gas pressure variations than the bulk migromegas due to the smaller amplification gap. This stability has been observed, over 30 days, during the operation in CAST as shown in figure 9 (right).

The Microbulk detectors showed the lowest background level among all detectors used in CAST, reaching less than $10^{-6} \mathrm{~s}^{-1} \mathrm{~cm}^{-2} \mathrm{keV}^{-1}$. Beyond this nominal operation, there have been several periods where the background have been reduced to the extraordinary low level of $\sim 2 \times 10^{-7} \mathrm{~s}^{-1} \mathrm{~cm}^{-2} \mathrm{keV}^{-1}[14,15]$, due to reasons which are under investigation, and which could be partially linked to variations in the radon concentration inside the shielding.

\subsection{Microbulk for NEXT}

NEXT [13] is a neutrinoless double beta decay experiment using a high pressure gas xenon TPC. Critical requirements for the detector in such an experiment is excellent energy resolution and background reduction from event topology, characteristics that has already been shown that the Microbulk meets.

In order to investigate the performance of Microbulks in high gas pressures, a series of experimental tests were performed and are still going on, at the Zaragoza University and at CEA Saclay. These tests have shown that there is no critical limitation for Micromegas to work up to 10 bar. No significant differences have been found in the response on low energy X-rays or high energy alpha particles, regarding the ionization yield or electronic transparency of Micromegas' mesh. Less than 1\% FWHM is pointed out as the intrinsic energy resolution of Micromegas for $5.5 \mathrm{MeV}$ alphas in argon-isobutane mixtures. There was no indication in these tests that these results have to be strongly dependent on pressure (probed up to 4.5 bar) or gas mixture (Microbulk have run in xenon without quencher). A detailed report on these results can be found at [8].

Another development towards a large scale TPC was the successful testing of the first 10x10 $\mathrm{cm}$ Microbulk with pixelised readout. The first medium area $(30 \times 30 \mathrm{~cm})$ Microbulk is currently being built.

\subsection{Microbulk in $\mathbf{n}_{-}$TOF}

The $\mathrm{n}_{-} \mathrm{TOF}$ experiment [16]-[17] is performing high precision cross-section measurements of neutron-induced reactions, using a neutron beam of a very wide energy range (thermal up to GeV). A critical point in these measurements is the precise knowledge of the energy distribution of the neutron flux at the measured sample position, without the need to measure the absolute flux. In the ideal case the flux measurements should be performed in parallel with the cross-section ones. Therefore a very low mass detector is needed to minimize the beam distraction and the background induced to the other detectors. Furthermore, the detector should show high resistance to radiation and low gamma sensitivity. A system consisting of a ${ }^{6} \mathrm{Li}$ deposit on a mylar foil and 4 offbeam silicon detectors detecting the ${ }^{6} \mathrm{Li}(\mathrm{n}, \alpha)^{3} \mathrm{H}$ reaction products has been used [18], Although the ${ }^{6} \mathrm{Li}(\mathrm{n}, \alpha)^{3} \mathrm{H}$ total cross section is considered a standard, the angle differential cross section is not. Therefore the useable energy range of this detector is limited by the uncertainties in the angular distribution. In addition the angular efficiency of the detector is difficult to measure.

A detector system based on Microbulk Micromegas was designed (figure 10 left) in order to be placed in the neutron beam in a short distance in front of the measuring point. The system consists 



Figure 10. Left: schematic layout of the Microbulk based neutron flux monitor. Right: amplitude spectrum of ${ }^{10} B(n, \alpha)$ reaction obtained at $\mathrm{n}_{-}$TOF facility.

of two Micromegas detectors placed inside a cylindrical chamber closed at the ends by kapton foils of $75 \mu \mathrm{m}$ thick facing ${ }^{10} \mathrm{~B}$ and ${ }^{235} \mathrm{U}$ neutron converters. In case the detector is placed in air the thickness of the kapton can be reduced to $10 \mu \mathrm{m}$. The detector consists of a drift cathode of 1.5 $\mu \mathrm{m}$ aluminized mylar with $1 \mathrm{mg}$ of ${ }^{10} \mathrm{~B}$, and a second drift cathode of $1.5 \mu \mathrm{m}$ aluminized mylar with $1 \mathrm{mg}$ of ${ }^{235} \mathrm{U}$. Each Microbulk has two $5 \mu \mathrm{m}$ thick copper layers and $25 \mu \mathrm{m}$ kapton forming the pillars. The pillars were constructed with a pitch of $500 \mu \mathrm{m}$, minimizing thus the material to the point that the main disturbance to the beam is caused by the neutron converters. In parallel, such a detector consisting of kapton and copper only shows very high radiation resistance.

The detector was used in laboratory and beam tests and was installed in $\mathrm{n}_{-} \mathrm{TOF}$ during the commissioning and start-up measurements in 2009. It showed the good energy resolution of all Microbulks, allowing the separation of the reaction fragments (alphas and ${ }^{7} \mathrm{Li}$ for ${ }^{10} \mathrm{~B}$, fission fragments and alphas for ${ }^{235} \mathrm{U}$, figure 10 right), while it has been insensitive to the intense gamma-flash that accompanies every neutron pulse in $\mathrm{n}_{-} \mathrm{TOF}$. Its performance was very stable, without major disturbance on the other measurements, and the system will be used for the rest of the campaign. Furthermore, the segmentation of the micromesh will allow the construction of a low mass detector with "real" 2D readout scheme for the first time, to be used as flux and beam profile monitor.

\section{Future developments}

The capacity of the detector is higher in the first fabrication mode described in section 2 because of the large quantity of kapton left in between the electrodes. We will push the etching process in order to remove as much kapton as possible and leave only tiny pillars. This improvement is necessary not only for lowering the electronic noise but also for decreasing the amount of charge released during an occasional discharge.

Up to now two-dimensional strip read-out in previous Micromegas detectors was possible by interconnecting pads in the anode plane as explained in the previous section. The new micro-Bulk technology offers the possibility to segment the cathode element, the copper mesh. Such X-Y read-out is suitable in some applications where a fully correlated anode-cathode signal is required. 


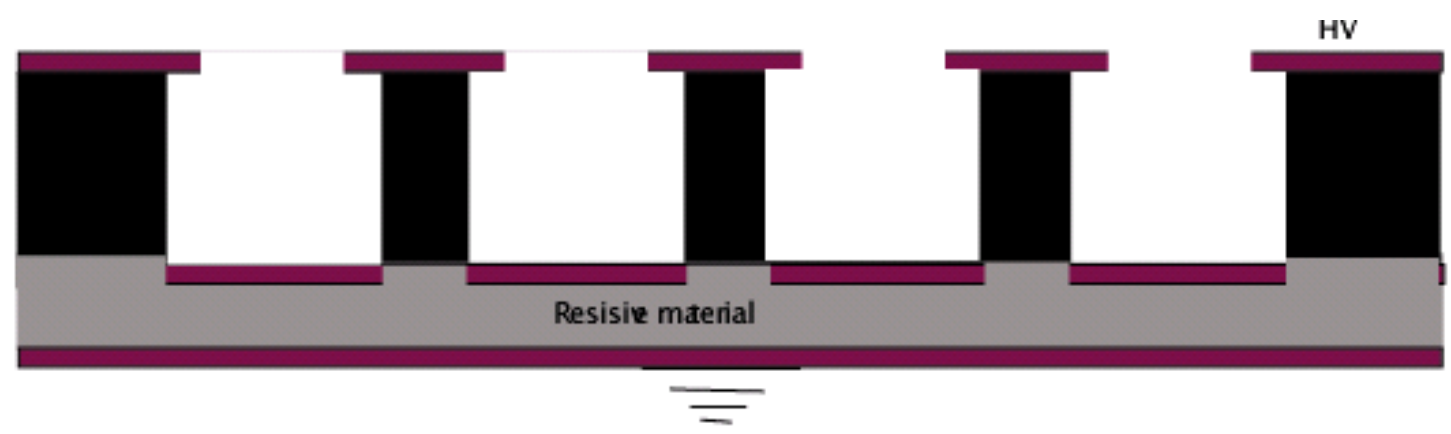

Figure 11. In red from top to bottom the copper mesh, the pillars, the bottom copper pads, the resistive layer, the bottom copper plane.

The size of the Microbulk will also be pushed as much as possible to tackle some applications requiring large detector area. To be able to produce larger size detectors we will follow also new developments going on at CERN through the RD51 collaboration. A study is going on to upgrade the equipment and the organization of the existing facility and contacts with industrial partners could also help to reach this goal.

For applications requiring operation at high pressures we are interested in decreasing the amplification gap. Present technology allows to fabricate $25 \mu \mathrm{m}$ gap and our next goal is a $12.5 \mathrm{~mm}$ gap on a commercially available kapton foil. However such development requires a simultaneous decrease of the mesh openings and hole pitch. In order to achieve holes as low as $15 \mu \mathrm{m}$ and pitches of $30 \mu \mathrm{m}$ we need to change the mask technology for the photolithography process. A precise mask made out of glass, available in the industry, should be developed. Another idea is to interface the micro-Bulk structure to the read-out strip through a resistive thin layer as shown in figure 11. Using lithographic process the bottom copper is forming small circular pads facing the holes of the micromesh and then a resistive glue or resistive paste is used to interconnect the micro-Bulk to the read-out plane (presented by the copper layer in the drawing).

\section{Conclusion}

The new micro-Bulk Micromegas is a quite successful technique used already by some experiments. It provides excellent uniformity and energy resolution and could be manufactured by an industrial process. The detector has low material budget and could provide cylindrical or other curved shapes.Its low intrinsic radioactivity and stability make this detector quite attractive for low background rare event search. For high rate particle physics experiment the detector could be used as internal high-precision tracker. Its low material is a key point to achieve good momentum resolution in high energy physics spectrometers, usually limited by the effect of multiple scattering of charged particles in the detector material

\section{References}

[1] Y. Giomataris, P. Rebourgeard, J.P. Robert and G. Charpak, MicrOMEGAs: a high-granularity position-sensitive gaseous detector for high particle-flux environments, Nucl. Instrum. Meth. A 376 (1996) 29. 
[2] Y. Giomataris, Development and prospects of the new gaseous detector 'MicrOMEGAs', Nucl. Instrum. Meth. A 419 (1998) 239.

[3] G. Charpak, J. Derre, Y. Giomataris and P. Rebourgeard, MicrOMEGAs, a multipurpose gaseous detector, Nucl. Instrum. Meth. A 478 (2002) 26.

[4] A. Delbart et al., New developments of MicrOMEGAs detector, Nucl. Instrum. Meth. A 461 (2001) 84.

[5] I. Giomataris et al., MicrOMEGAs in a bulk, Nucl. Instrum. Meth. A 560 (2006) 405 [physics/0501003].

[6] F. Sauli, GEM: a new concept for electron amplification in gas detectors, Nucl. Instrum. Meth. A 386 (1997) 531.

[7] T. Dafni et al., Energy resolution of alpha particles in a microbulk MicrOMEGAs detector at high pressure Argon and Xenon mixtures, Nucl. Instrum. Meth. A 608 (2009) 259.

[8] A. Tomas et al, Development of Micromegas towards Double Beta Neutrinoless Decay Searches, $1^{\text {st }}$ International Conference on Micro Pattern Gaseous Detectors, June 12-15, 2009, Kolympari, Crete, Greece.

[9] CAST collaboration, K. Zioutas et al., First results from the CERN Axion Solar Telescope (CAST), Phys. Rev. Lett. 94 (2005) 121301 [hep-ex/0411033].

[10] CAST collaboration, S. Andriamonje et al., An improved limit on the axion-photon coupling from the CAST experiment, JCAP 04 (2007) 010 [hep-ex/0702006].

[11] CAST collaboration, E. Arik et al., Probing eV-scale axions with CAST, JCAP 02 (2009) 008 [arXiv:0810.4482].

[12] P. Abbon et al., The Micromegas detector of the CAST experiment, New J. Phys. 9 (2007) 170.

[13] THE NEXT collaboration, . F. Granena et al., NEXT, a HPGXe TPC for neutrinoless double beta decay searches, arXiv:0907.4054.

[14] S. Aune et al., An ultra-low-background detector for axion searches, J. Phys. Conf. Ser. 179 (2009) 012015.

[15] J. Galan et al, Micromegas Detectors in the CAST Experiment, $1^{\text {st }}$ International Conference on Micro Pattern Gaseous Detectors, June 12-15, 2009, Kolympari, Crete, Greece.

[16] C. Rubbia et al., A High Resolution Spallation Driven Facility at the CERN-PS to Measure Neutron Cross Sections in the Interval from $1 \mathrm{eV}$ to $250 \mathrm{MeV}$ : a Relative Performance Assessment, CERN-LHC-98-002-EET-Add.1 (1998).

[17] $\mathrm{n}_{-}$TOF collaboration F. Gunsing et al., Status and outlook of the neutron time-of-fight facility $n_{-}$TOF at CERN, Nucl. Instrum. Meth. B 261 (2007) 925.

[18] S. Marrone et al., A low background neutron flux monitor for the $n_{-}$TOF facility at CERN, Nucl. Instrum. Meth. A 517 (2004) 389. 